\title{
Bonding, vibrational, and electrical characteristics of CdS nanostructures embedded in polyvinyl alcohol matrix
}

\author{
S. P. Mondal, ${ }^{1}$ A. Dhar, ${ }^{1}$ S. K. Ray, ${ }^{1, a)}$ and A. K. Chakraborty ${ }^{2}$ \\ ${ }^{1}$ Department of Physics and Meteorology, Indian Institute of Technology, Kharagpur-721 302, India \\ ${ }^{2}$ Laboratory for Mechanical Systems Engineering EMPA, Duebendorf, Zurich, CH-8600, Switzerland
}

(Received 17 September 2008; accepted 1 March 2009; published online 23 April 2009)

CdS nanocomposites have been grown in polyvinyl alcohol matrix by a chemical synthesis process. The transmission electron micrographs of nanocomposites synthesized at $70-90{ }^{\circ} \mathrm{C}$ temperature showed the growth of needlelike and junctionlike nanostructures. X-ray photoelectron spectroscopy analysis revealed the growth of stoichiometric $\mathrm{CdS}$ without the formation of any intermediate phases at the CdS-polyvinyl alcohol interface. Raman spectra of first order longitudinal optical phonon peak has been analyzed using phonon dispersion model to detect the surface phonon modes in $\mathrm{CdS}$ nanoneedles and wires. The origin of negative differential resistance behavior in current-voltage characteristics for junctionlike CdS nanocomposites has been discussed. () 2009 American Institute of Physics. [DOI: 10.1063/1.3111971]

\section{INTRODUCTION}

Polymer inorganic-semiconductor nanocomposites have attracted much attention recently due to their unique sizedependent chemical and physical properties. ${ }^{1-5}$ Incorporation of zero dimensional and one dimensional nanostructures such as $Q$-dots and nanowires/nanotubes in polymer matrix is expected to enhance the functionality of composites. A lot of investigations have been carried out on the synthesis of low dimensional semiconductor nanostructures embedded in conducting as well as insulating polymer matrices for their possible applications in memories, ${ }^{6,7}$ light emitting diodes, ${ }^{8}$ high efficiency solar cells, ${ }^{9}$ and different sensing devices. ${ }^{10}$ CdS nanostructures embedded in polymer matrix have been reported to exhibit excellent optical as well as electrical characteristics, ${ }^{6,7}$ which are attractive not only for fundamental physics but also for their possible applications in the next generation organic and flexible electronics. We have reported the formation of one dimensional junctionlike $\mathrm{CdS}$ nanostructures inside polyvinyl alcohol (PVA) with shapes varying from needle to wire with the increase in bath temperature. The local chemical bonding and semiconductor/ polymer interface play a crucial role in determining the optical and electrical properties of nanocomposites. A detailed x-ray photoelectron spectroscopy (XPS) study has been carried for chemical analysis of the nanocomposite films. The paper presents the bonding, vibrational, and electrical characteristics of CdS-PVA nanocomposites grown at different bath temperatures. The origin of surface phonon modes in Raman spectra and negative resistance behavior in junctionlike nanocomposites is discussed.

\section{EXPERIMENTAL}

CdS nanocomposites were synthesized in polyvinyl alcohol matrix using a simple chemical bath deposition (CBD) technique. ${ }^{11}$ The nanocomposite films were grown on (100)

${ }^{a)}$ Electronic mail: physkr@phy.iitkgp.ernet.in.
$p$-Si $(7-14 \Omega \mathrm{cm})$ and commercially available indium tin oxide (ITO) coated glass substrates at different bath temperatures from $70-90^{\circ} \mathrm{C}$. A thick CdS film, as a control sample, was also deposited on ITO coated glass substrates by a CBD process. Microstructural studies of deposited films were performed using a transmission electron microscope (JEOL JEM-2100F) with an accelerating voltage of $200 \mathrm{kV}$. XPS measurements were carried out in an ultrahigh vacuum chamber of a Scienta ESCA 300 photoelectron spectrometer with a base pressure of $1 \times 10^{-6} \mathrm{mbar}$. The spectra were recorded at normal emission with $\mathrm{Al} \mathrm{K} \alpha$ excitation ( $h v$ $=1486.7 \mathrm{eV}$ ) take off angle $45^{\circ}$ and an energy resolution of $0.4 \mathrm{eV}$. The energy was calibrated using C $1 s$ (at $285 \mathrm{eV}$ ) of adventitious carbon as a reference. Raman spectroscopy of the nanocomposites was studied using a JASCO Corp., NRS1000 system (power $0.1 \mathrm{~mW}$ and spot size $100 \mu \mathrm{m}$ ) with a laser of wavelength $532 \mathrm{~nm}$. Aluminum electrodes of area $3.14 \times 10^{-6} \mathrm{~m}^{2}$ were thermally evaporated on nanocomposites and control films for electrical contacts. The current voltage characteristics of the films were measured using a programmable Keithley-485 picoammeter and Advantest-R6144 constant dc voltage source.

\section{RESULT AND DISCUSSIONS}

Figure 1 shows the plane-view transmission electron micrographs (TEM) of $\mathrm{CdS}$ nanocomposite films grown at $70-90{ }^{\circ} \mathrm{C}$ bath temperature in PVA matrix. The micrographs clearly demonstrate the formation of isolated needlelike structures at $70{ }^{\circ} \mathrm{C}$ [Fig. 1(a)], $Y$ - and $T$-junctionlike nanostructures at $80^{\circ} \mathrm{C}$ [Fig. 1(b)] and junctionlike nanowires at $90{ }^{\circ} \mathrm{C}$ [Fig. 1(c)]. From the TEM micrographs, it is evident that as the bath temperature increases from 70 to $90{ }^{\circ} \mathrm{C}$, the structure changes from isolated $\mathrm{CdS}$ nanoneedles to branched nanowires. The temperature dependent growth mechanism, optical, and dielectric properties of junctionlike $\mathrm{CdS}$ nanostructures have been discussed in detail in our previous paper. ${ }^{11}$ 


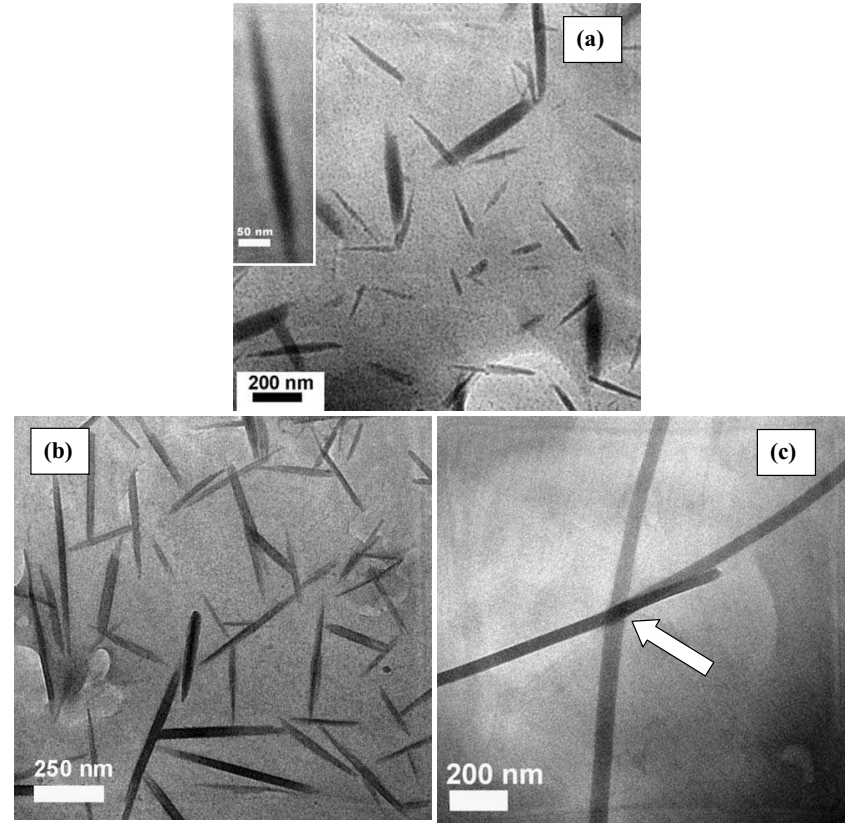

FIG. 1. Plane-view TEM micrographs of CdS nanocomposites grown at different temperatures (a) nanoneedles at $70^{\circ} \mathrm{C}$. Inset represents a single nanoneedle of average diameter $\sim 40 \mathrm{~nm}$ and length $\sim 400 \mathrm{~nm}$. (b) Junctionlike structures at $80{ }^{\circ} \mathrm{C}$ and (d) a wirelike $Y$-junction at $90{ }^{\circ} \mathrm{C}$.

To investigate the chemical bonding of the nanocomposite samples, XPS was carried out at room temperature. The XPS survey scan of CdS-PVA nanocomposites grown at 70, 80,85 , and $90{ }^{\circ} \mathrm{C}$ bath temperatures is depicted in Fig. 2. At all growth temperatures, the elements $\mathrm{Cd}, \mathrm{S}, \mathrm{Cl}, \mathrm{N}, \mathrm{C}$, and $\mathrm{O}$ are detected. The dominant source of carbon and oxygen impurities in nanocomposites is originated from the host matrix polyvinyl alcohol. Thiourea $\left[\left(\mathrm{NH}_{2}\right)_{2} \mathrm{CS}\right]$, which was used in chemical reaction, may also be a potential source of C. The chlorine concentration in as-deposited $\mathrm{CdS}$ is due to the reagents $\mathrm{CdCl}_{2}$ and $\mathrm{NH}_{4} \mathrm{Cl}$ used for the preparation of $\mathrm{CdS}$ nanocomposites by $\mathrm{CBD}$ process. The nitrogen peak may be attributed to the urea $\left[\left(\mathrm{NH}_{2}\right)_{2} \mathrm{CO}\right]$ formation during the chemical reaction process. ${ }^{12}$ Figure 3 presents the binding energy of $\mathrm{Cd} 3 d$ photoelectrons of the nanocomposites grown at different temperatures. Each spectrum exhibits a

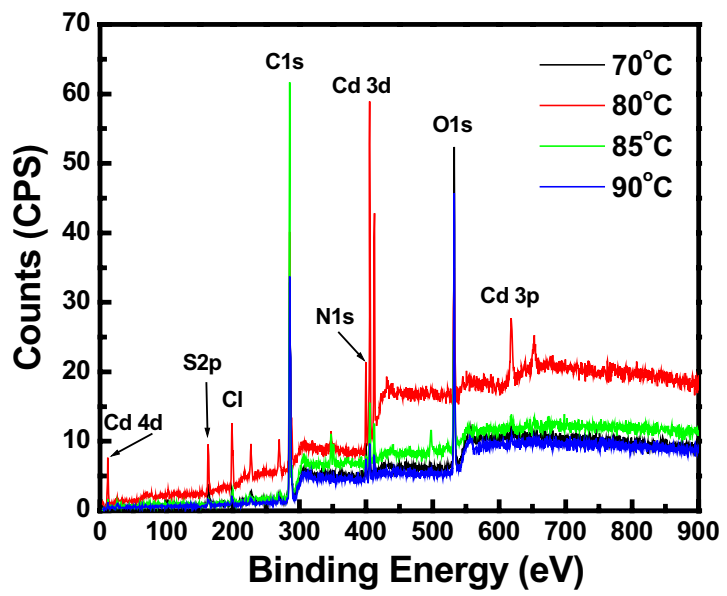

FIG. 2. (Color online) XPS survey scan of CdS nanocomposites grown at $70,80,85$, and $90{ }^{\circ} \mathrm{C}$ growth temperature.

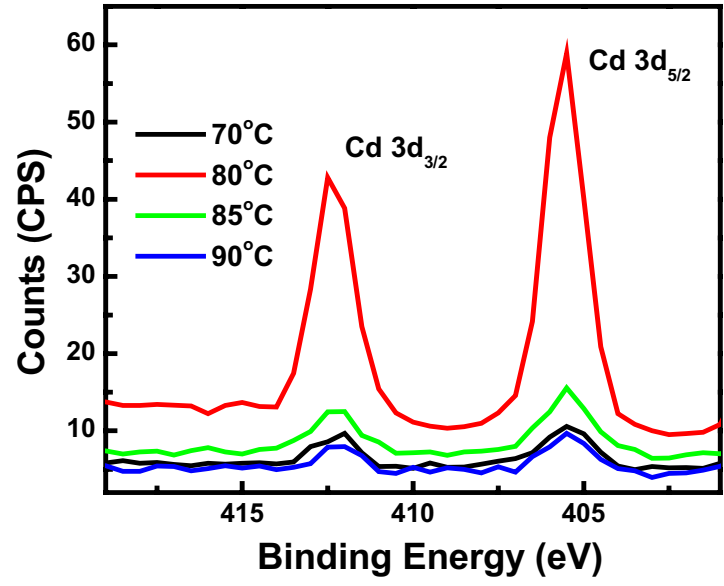

FIG. 3. (Color online) High resolution XPS spectra of Cd $3 d$ energy level of the nanocomposites.

doublet feature due to spin-orbit splitting resulting into $3 d_{3 / 2}$ and $3 d_{5 / 2}$ peaks with a spin-orbit separation of $7.0 \mathrm{eV}$. The binding energy of $3 d_{5 / 2}$ and $3 d_{3 / 2}$ electrons of CdS nanocomposites is found to be 405.5 and $412.5 \mathrm{eV}$, respectively, which correspond to $\mathrm{Cd}$ in stoichiometric $\mathrm{CdS} .{ }^{13}$ Figure 4 shows the sulfur $2 p$ spectrum of the nanocomposites, with inset representing the typical fitted spectrum of $\mathrm{S} 2 p$ photoelectrons. The spectrum is deconvoluted into two Gaussian peaks, which are attributed to $\mathrm{S} 2 p_{1 / 2}$ and $\mathrm{S} 2 p_{3 / 2}$ levels due to spin-orbit interaction. For the $80{ }^{\circ} \mathrm{C}$ grown sample the $\mathrm{S} 2 p_{1 / 2}$ and $\mathrm{S} 2 p_{3 / 2}$ levels are situated at 163.0 and $162.4 \mathrm{eV}$, which corresponds to $\mathrm{S}$ comes from CdS. ${ }^{14}$ The absence of any intermediate phases at the semiconductor-PVA interface is apparent from the XPS analysis of Cd $3 d$ and S $2 p$ spectra. The chemical composition also remains the same in the studied growth temperature range. The results indicate that the PVA matrix does not alter the local chemical bonding in $\mathrm{CdS}$ nanostructures, which is an essential requirement for functional materials using inorganic-organic heterostructures.

Figure 5 shows the valence band spectra of CdS-PVA nanocomposites grown at 70 and $80^{\circ} \mathrm{C}$. The photoemission spectra of the nanocomposites is similar to the bulk $\mathrm{CdS}$

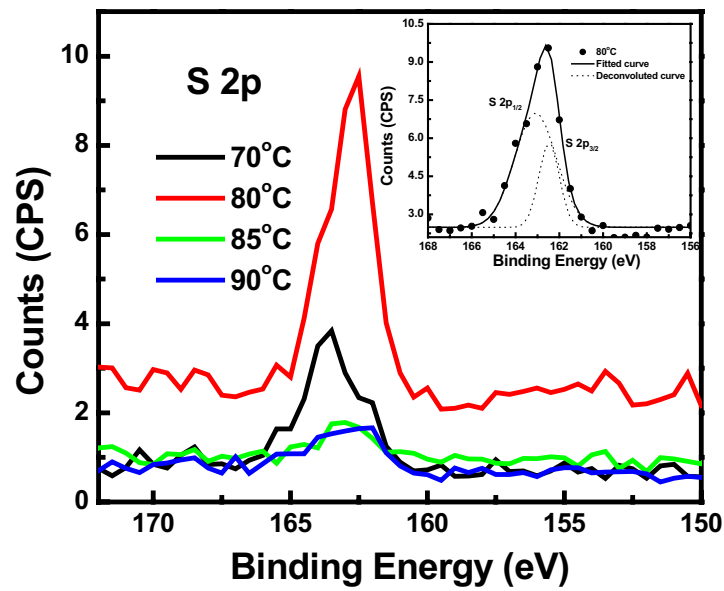

FIG. 4. (Color online) High resolution XPS spectra of S $2 p$ energy level of CdS-PVA nanocomposites grown at different temperatures. Inset figure represents the typical fitted spectrum of S $2 p$ energy level of the nanocomposite grown at $80{ }^{\circ} \mathrm{C}$. 


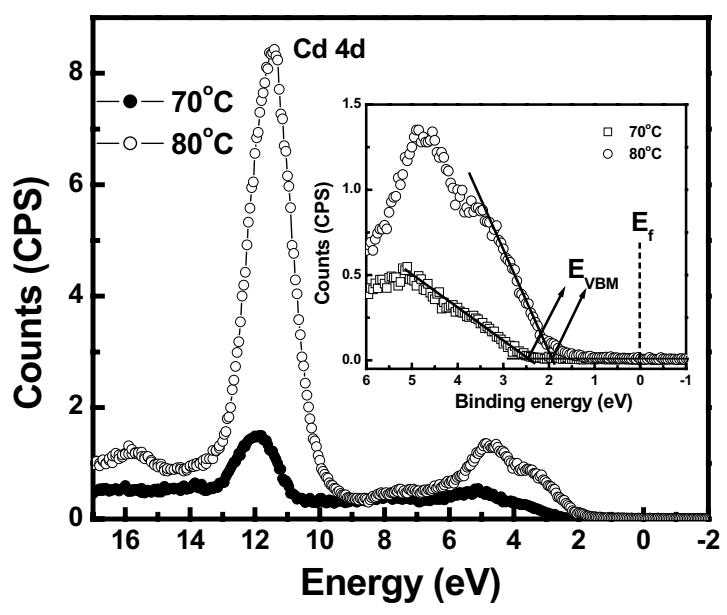

FIG. 5. XPS valence band spectra of the nanocomposite samples grown at 70 and $80{ }^{\circ} \mathrm{C}$ temperature. Inset shows the position of valence band for 70 and $80{ }^{\circ} \mathrm{C}$ temperature growth samples.

data. ${ }^{15}$ The peak at $11.4-11.9 \mathrm{eV}$ is assigned to the shallow $4 d$ cadmium core, whereas the broadened peak at $\sim 5 \mathrm{eV}$ is due to the valence band of the nanocomposites. The position of the valence band maxima $\left(E_{\mathrm{VBM}}\right)$ has been determined by linear extrapolation of the leading edge to the energy axis. The value of $E_{\mathrm{VBM}}$ for the nanocomposites synthesized at 70 and $80{ }^{\circ} \mathrm{C}$ is found to be 2.4 and $1.9 \mathrm{eV}$, respectively. It is clear from the inset of the Fig. 5 that the $E_{\mathrm{VBM}}$ is shifted to the lower energy value at a higher growth temperature. We observed $^{11}$ an increase in optical band gap energy of the nanocomposites with decreasing growth temperatures, due to the formation of smaller crystallites. Therefore, the shift in valence band edge toward higher energy may be attributed to reduce size of CdS clusters at lower growth temperature, in agreement with the reported results. ${ }^{16}$

Figure 6 shows the Raman spectra of CdS-PVA nanocomposites grown at different temperatures. Raman peaks at 300,601 , and $907 \mathrm{~cm}^{-1}$, respectively, are attributed to the first, second, and third order longitudinal optical (LO) phonon vibrational modes of CdS. While the first two modes are

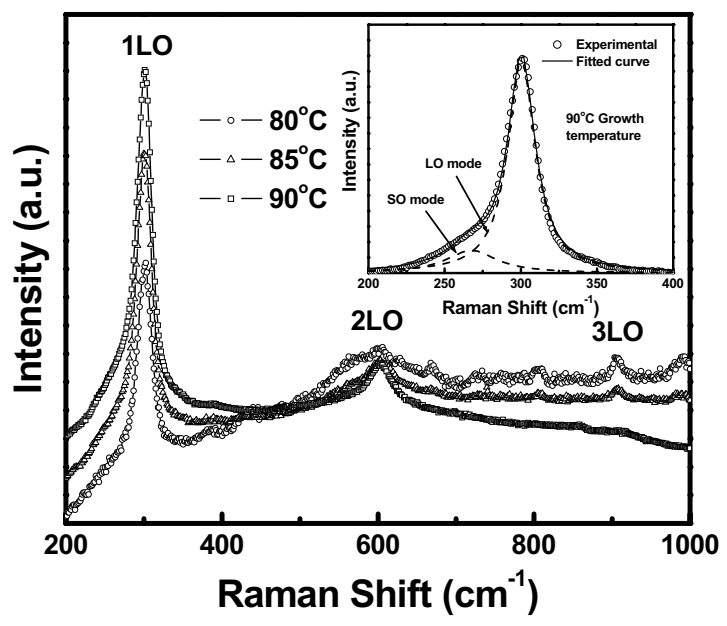

FIG. 6. Raman spectra of CdS nanocomposites at different growth temperatures. Inset shows $1 \mathrm{LO}$ peak deconvoluted by two Lorentzians for the $90{ }^{\circ} \mathrm{C}$ sample. Circles represent experimental points, solid line fitted to the data, and dashed lines represent individual Raman modes. prominent, the third order LO peak is relatively weak. However, as the growth temperature increases, the Raman peak broadening is reduced and intensity is enhanced due to improved crystallinity in corroboration with our reported x-ray diffraction results. ${ }^{11}$ The most interesting feature of the Raman spectra is the presence of an asymmetry in the first order LO peak at lower frequency region. It is well known that in a crystalline insulator or semiconductor, the observed Raman shifts usually correspond to the LO phonons; whereas the transverse optic (TO) and the surface optical (SO) phonon modes are suppressed because of the symmetry restrictions and their low intensities, respectively. However, in case of nanostructures, the quantum size effects ${ }^{17,18}$ as well as enhanced surface-to-volume ratio make the appearance of SO mode plausible. ${ }^{19}$ The SO phonon mode in nanocrystals appears in between the $\left(\omega_{\mathrm{LO}}\right)$ and $\left(\omega_{\mathrm{TO}}\right)$ phonon frequencies, resulting in an asymmetry in $1 \mathrm{LO}$ peak. ${ }^{20}$ The SO phonon mode peak has been extracted by deconvoluting the characteristic 1LO peak with two Lorentzians, having integrated intensity given by

$$
I(\omega)=\frac{A_{\mathrm{SO}} \omega \Gamma_{\mathrm{SO}}}{\left(\omega_{\mathrm{SO}}^{2}-\omega^{2}\right)^{2}+\left(\Gamma_{\mathrm{SO}} \omega\right)^{2}}+\frac{A_{\mathrm{LO}} \omega \Gamma_{\mathrm{LO}}}{\left(\omega_{\mathrm{LO}}^{2}-\omega^{2}\right)^{2}+\left(\Gamma_{\mathrm{LO}} \omega\right)^{2}},
$$

where $A_{\mathrm{SO}}\left(A_{\mathrm{LO}}\right), \omega_{\mathrm{SO}}\left(\omega_{\mathrm{LO}}\right)$, and $\Gamma_{\mathrm{SO}}\left(\Gamma_{\mathrm{LO}}\right)$ are the oscillator strength, frequency, and line width of the modes corresponding to the shoulder (LO phonon), respectively. The parameters $A_{\mathrm{LO}}, \omega_{\mathrm{LO}}$, and $\Gamma_{\mathrm{LO}}$ have been extracted by fitting two Lorentzians to the experimental data points. A typical fitted spectrum for the $90{ }^{\circ} \mathrm{C}$ grown sample is shown in the inset of Fig. 6 with the fitted curve to experimental points represented by the solid line, and dashed lines representing spectrum corresponding to individual modes. To assign the SO phonon mode, we consider the dispersion relation $\omega_{\mathrm{SO}}(q)$ for an infinite long cylindrical wire, in the limit where the phonon wave vector $q \gg \omega / c$ (Refs. 21 and 22),

$$
\begin{aligned}
& \omega_{\mathrm{SO}}^{2}=\omega_{\mathrm{TO}}^{2}+\frac{\omega_{p}^{2}}{\varepsilon_{\infty}+\varepsilon_{m} f(x)} ; \\
& x=q r,
\end{aligned}
$$

where $\omega_{\mathrm{TO}}$ is the TO mode frequency at the zone center, $\omega_{p}$ is the screened ion plasma frequency given by

$$
\omega_{\mathrm{LO}}^{2}=\omega_{\mathrm{TO}}^{2}+\frac{\omega_{p}^{2}}{\varepsilon_{\infty}} .
$$

$\varepsilon_{\infty}$ and $\varepsilon_{m}$ are the high-frequency dielectric constant of bulk $\mathrm{CdS}$ and host matrix (PVA), respectively, $r$ the wire radius and $f(x)$ is obtained from the eigenvalue equation,

$$
f(x)=\frac{I_{0}(x) K_{1}(x)}{I_{1}(x) K_{0}(x)},
$$

where $I_{j}(x)$ and $K_{j}(x)$ are Bessel functions.

The TO phonon frequency of the nanocomposites has been calculated using Lyddane-Sachs-Teller relation, ${ }^{23}$ 
TABLE I. Surface phonon mode $\left(\omega_{\mathrm{SO}}\right)$ and LO phonon $\left(\omega_{\mathrm{LO}}\right)$ peak position at different growth temperatures. The peak positions have been extracted from the fitted parameters.

\begin{tabular}{ccccc}
\hline \hline $\begin{array}{c}\text { Growth } \\
\text { Temperature }\end{array}$ & $\begin{array}{c}\omega_{\mathrm{SO}} \mathrm{cm}^{-1} \\
\text { Extracted } \\
\text { values }\end{array}$ & $\begin{array}{c}\omega_{\mathrm{LO}} \mathrm{cm}^{-1} \\
\text { Extracted } \\
\text { values }\end{array}$ & $\begin{array}{c}\omega_{\mathrm{sO}} \mathrm{cm}^{-1}(x \sim 4) \\
\text { Calculated } \\
\text { values }\end{array}$ & $\begin{array}{c}\text { Intensity } \\
I_{\mathrm{SO}} / I_{\mathrm{LO}}\end{array}$ \\
\hline $80{ }^{\circ} \mathrm{C}$ & $257.2 \pm 1.4$ & $300.3 \pm 0.1$ & $\varepsilon_{m}=7 \omega_{s o}=257.1$ & 0.08 \\
$85{ }^{\circ} \mathrm{C}$ & $262.1 \pm 1.3$ & $300.2 \pm 0.1$ & $\varepsilon_{m}=5.2 \omega_{s o}=262.3$ & 0.09 \\
$90{ }^{\circ} \mathrm{C}$ & $267.6 \pm 1.4$ & $300.4 \pm 0.1$ & $\varepsilon_{m}=5.0 \omega_{s o}=263.0$ & 0.11 \\
\hline \hline
\end{tabular}

$$
\frac{\omega_{\mathrm{TO}}^{2}}{\omega_{\mathrm{LO}}^{2}}=\frac{\varepsilon_{\infty}}{\varepsilon_{l}} .
$$

The high and low frequency dielectric constants of CdS are taken as $\varepsilon_{\infty}=5.2$ and $\varepsilon_{l}=8.9$, respectively, whereas $\varepsilon_{m}$ of the dielectric constant of polyvinyl alcohol are varied from 5 to $8 .^{24,25}$ The calculated values of SO phonon frequencies $\left(\omega_{\mathrm{SO}}\right)$ at different growth temperatures are summarized in Table I. Using the $1 \mathrm{LO}$ peak of nanocomposites from Fig. 6 and Eqs. (2) $-(5)$, SO phonon frequencies $\left(\omega_{\mathrm{SO}}, x \sim 4\right)$ are found to be at $257.1,262.3$, and $263.0 \mathrm{~cm}^{-1}$ at growth temperatures of 80 , 85, and $90{ }^{\circ} \mathrm{C}$, respectively. This is an excellent agreement with the extracted $\omega_{\text {SO }}$ of 257.2 and 262.1 for nanocomposites grown at 80 and $85{ }^{\circ} \mathrm{C}$, respectively. The deviation of extracted $\omega_{\mathrm{SO}}\left(\sim 267.6 \mathrm{~cm}^{-1}\right)$ from the calculated one at $90{ }^{\circ} \mathrm{C}$ may be due to the modified dielectric characteristics of PVA, which depends on molecular weight, degree of polymerization, voids, discontinuities, and temperature. However, the intensity ratio of $\mathrm{SO}$ to $\mathrm{LO}$ mode $I_{\mathrm{SO}} / I_{\mathrm{LO}}$ (Table I) shows increasing nature with growth temperature.

Figures 7 and 8 show $I$ - $V$ characteristics of nanocomposites deposited on ITO coated glass and $p$-Si substrates, respectively. Interestingly, a negative differential resistance (NDR) behavior is clearly observed at a certain bias voltage in both the samples. Inset of Fig. 7 represents the $I-V$ characteristics of control CdS thin film deposited on ITO substrate without any NDR characteristics. This type of negative resistance behavior in $I-V$ characteristics at room temperature has been reported in thinner $(<10 \mathrm{~nm})$ composite polymeric films. ${ }^{26}$ However, in our case the origin of the negative resistance behavior is attributed to the tunneling between the

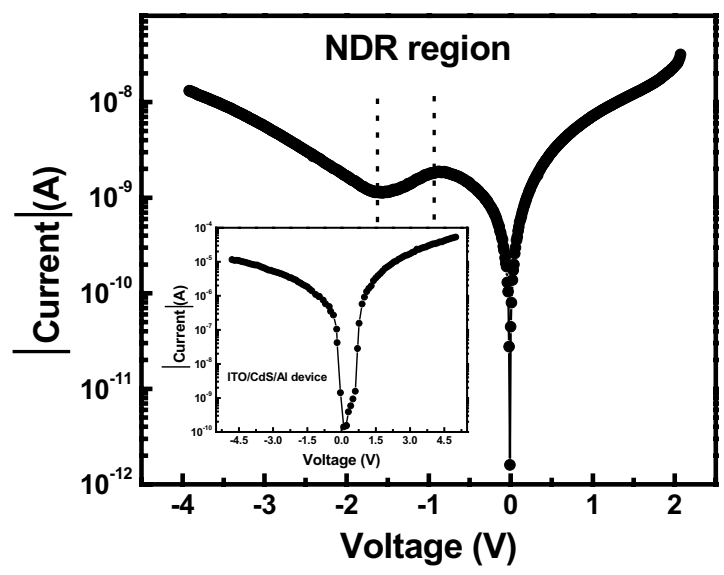

FIG. 7. Current-voltage characteristics of ITO/CdS nanocomposites/Al junction. The characteristics for control CdS film is shown in the inset.

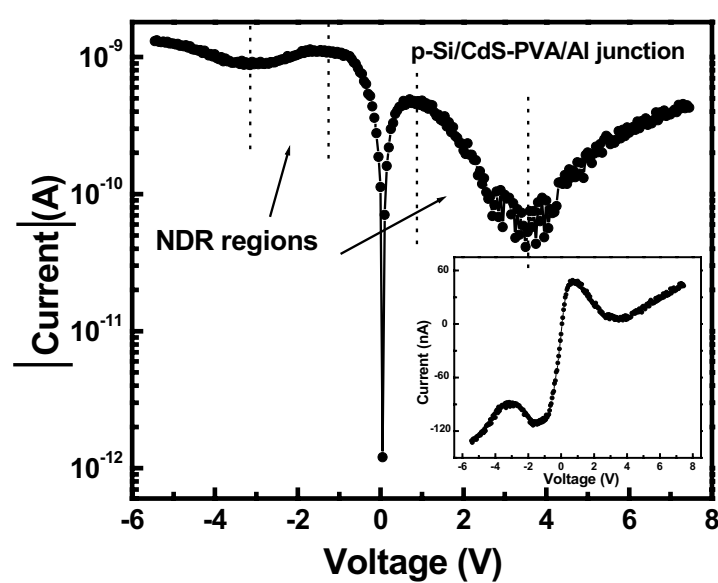

FIG. 8. $I-V$ characteristics of $p$-Si/CdS nanocomposites/Al junction. The plot in NDR region in a linear current scale is shown in the inset.

overlapping CdS nanowires embedded in the PVA matrix in a region outlined in TEM micrograph [Fig. 1(c)]. When the gap between two wires is low enough, the tunneling may take place exhibiting the negative resistance characteristics. The peak-to-valley current $\left(I_{p} / I_{v}\right)$ and voltage ratio $\left(V_{p} / V_{v}\right)$ of the ITO/CdS-PVA/Al device is calculated to be 1.7 and 0.54 , respectively. The single negative resistance peak in Fig. 7 is due to the formation of a junction with a single tunneling barrier between two nanowires. On the other hand, the appearance of negative resistance characteristics in both the bias conditions in Fig. 8 indicates the formation of a double junction, giving rise to back-to-back tunnel diodes. ${ }^{27}$ This can only happen due to the formation of an additional tunnel junction between $n$-CdS/ $p$-Si with a thin insulating PVA barrier. The efficacy of the insulating PVA as a barrier layer is supported by the XPS analysis of the nanocomposites showing an excellent interface between CdS nanowires and PVA. One of the junctions is forward biased for any biasing condition resulting in negative resistance peaks in both directions, as shown in the inset of Fig. 8. The peak-to-valley current $\left(I_{p} / I_{v}\right)$ and voltage ratio $\left(V_{p} / V_{v}\right)$ of the $p$-Si/CdS-PVA/Al device in positive and negative bias are found to be $11.1,1.6$, and $0.24,0.41$, respectively.

\section{CONCLUSIONS}

In conclusion, we have grown junctionlike $\mathrm{CdS}$ nanostructures inside a polymer matrix using a simple chemical technique. The detailed XPS analysis indicated the absence of nonstoichiometric and intermediate phases at the semiconductor/PVA interface at different growth temperatures. The VBM shifts to a higher value with decreasing growth temperature due to smaller CdS nanoparticles in corroboration with the enhancement of optical band gap. The asymmetry in Raman spectra has been attributed to the presence of SO phonon mode in nanocomposites. The SO phonon peak for different growth temperatures agrees with the estimated ones using a phonon dispersion relation of a long cylindrical wire. The negative resistance behavior in $I-V$ characteristics signifies the tunneling phenomena between nanowire junctions with an insulating PVA barrier. 


\section{ACKNOWLEDGMENTS}

This work was supported in part by a NSTI project grant of Department of Science and Technology, Government of India. One of the authors (S.P.M) acknowledges the Council of Scientific and Industrial Research, Government of India, for awarding a fellowship to carry out this work.

${ }^{1}$ V. L. Colvin, M. C. Schlamp, and A. P. Alivisatos, Nature (London) 370, 354 (1994).

${ }^{2}$ S. Maeda and S. P. Armes, Chem. Mater. 7, 171 (1995).

${ }^{3}$ P. G. Hill, P. J. S. Foot, and R. Davis, Mater. Sci. Forum 191, 43 (1995).

${ }^{4}$ R. E. Schwerzel, K. B. Spahr, J. P. Kurmer, V. E. Wood, and J. A. Jenkins, J. Phys. Chem. A 102, 5622 (1998).

${ }^{5}$ T. Trindade, M. C. Neves, and A. M. V. Barros, Scr. Mater. 43, 567 (2000). ${ }^{6}$ S. P. Mondal, V. S. Reddy, S. Das, A. Dhar, and S. K. Ray, Nanotechnology 19, 215306 (2008).

${ }^{7}$ F. Li, D.-I. Son, H.-M. Cha, S.-M. Seo, B.-J. Kim, H.-J. Kim, J.-H. Jung, and T. W. Kim, Appl. Phys. Lett. 90, 222109 (2007).

${ }^{8}$ K. S. Narayan, A. G. Manoj, J. Nanda, and D. D. Sarma, Appl. Phys. Lett. 74, 871 (1999).

${ }^{9}$ L. Wang, Y. S. Liu, X. Jiang, D. H. Qin, and Y. Cao, J. Phys. Chem. C 111, 9538 (2007).

${ }^{10}$ S. A. Mcdonald, G. Konstantatos, S. Zhang, P. W. Cyr, E. J. D. Klem, L. Levina, and E. H. Sargent, Nature Mater. 4, 138 (2005).

${ }^{11}$ S. P. Mondal, H. Mullick, T. Lavanya, A. Dhar, S. K. Lahiri, and S. K.
Ray, J. Appl. Phys. 102, 064305 (2007).

${ }^{12}$ S. Kolhe, S. K. Kulkarni, A. S. Nigavekar, and V. G. Bhide, J. Mater. Sci. 22, 1067 (1987).

${ }^{13}$ M. Stoev and A. Katerski, J. Mater. Chem. 6, 377 (1996).

${ }^{14}$ J. Nanda, B. A. Kuruvilla, and D. D. Sarma, Phys. Rev. B 59, 7473 (1999).

${ }^{15}$ L. Ley, R. A. Pollak, F. R. McFeely, S. P. Kowalczyk, and D. A. Shirley, Phys. Rev. B 9, 600 (1974).

${ }^{16}$ V. L. Colvin and A. P. Alivisatos, Phys. Rev. Lett. 66, 2786 (1991).

${ }^{17}$ S. Hayashi, H. Sanda, M. Agata, and K. Yamamoto, Phys. Rev. B 40, 5544 (1989).

${ }^{18}$ I. H. Campbell and P. M. Fauchet, Solid State Commun. 58, 739 (1986).

${ }^{19}$ A. Mlayah, A. M. Brugman, R. Carles, J. B. Renucci, M. Ya. Valakh, and A. V. Pogorelov, Solid State Commun. 90, 567 (1994).

${ }^{20}$ Q. Xiong, J. Wang, O. Reese, L. C. Lew Yan Voon, and P. C. Eklund, Nano Lett. 4, 2004 (1991).

${ }^{21}$ B. E. Sernelius, Surface Modes in Physics (Wiley-VCH, New York, 2001).

${ }^{22}$ R. Gupta, Q. Xiong, G. D. Mahan, and P. C. Eklund, Nano Lett. 3, 1745 (2003).

${ }^{23}$ C. Kittel, Introduction to Solid State Physics (Wiley, New York, 1971).

${ }^{24}$ Y. Sun, Y. Liu, Y. Ma, C. Di, Y. Wang, W. Wu, G. Yu, W. Hu, and D. Zhu, Appl. Phys. Lett. 88, 242113 (2006).

${ }^{25}$ R. Schroeder, L. A. Majewski, and M. Grell, Adv. Mater. (Weinheim, Ger.) 17, 1535 (2005).

${ }^{26}$ N. Glezos, P. Argitis, D. Velessiotis, and C. D. Diakoumakos, Appl. Phys. Lett. 83, 488 (2003).

${ }^{27}$ M. Stoffel, G. S. Kar, and O. G. Schmidt, Mater. Sci. Eng., C 25, 826 (2005). 